\title{
A new update for radiocontrast-induced nephropathy aggravated with glycerol in rats: the protective potential of epigallocatechin-3-gallate
}

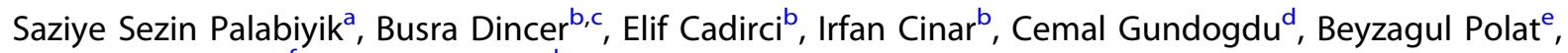 \\ Muhammed Yayla ${ }^{f}$ and Zekai Halici ${ }^{b}$ \\ ${ }^{\mathrm{a}}$ Faculty of Pharmacy, Pharmaceutical Toxicology Department, Ataturk University, Erzurum, Turkey; ${ }^{\mathrm{b}}$ Faculty of Medicine, Pharmacology \\ Department, Ataturk University, Erzurum, Turkey; ${ }^{C}$ Faculty of Pharmacy, Pharmacology Department, Erzincan University, Erzincan, \\ Turkey; ${ }^{\mathrm{d}}$ Faculty of Medicine, Pathology Department, Ataturk University, Erzurum, Turkey; ${ }^{\mathrm{e}}$ Faculty of Pharmacy, Pharmacology

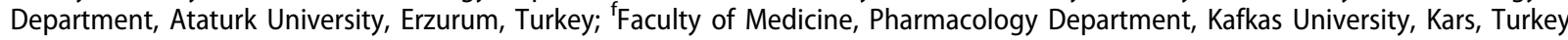

ABSTRACT

Contrast media (CM) is known to have nephrotoxic adverse effects. Epigallocatechin-3-gallate (EGCG) is the most abundant and active catechin in green tea, and has strong antioxidant and anti-inflammatory properties. This study investigated whether EGCG can reduce contrast-induced nephrotoxicity (CIN), alone or with glycerol (GLY)-induced renal damage, and to understand its mechanisms of protection against toxicity, using models of GLY and CIN in rats. The rats were separated into eight groups ( $n=6$ in each), as follows: Healthy, GLY, CM, GLY + CM, CM + EGCG $50 \mathrm{mg} / \mathrm{kg} \quad$ (po), GLY + CM + EGCG $50 \mathrm{mg} / \mathrm{kg} \quad$ (po), CM + EGCG $100 \mathrm{mg} / \mathrm{kg} \quad$ (po), and $\mathrm{GLY}+\mathrm{CM}+\mathrm{EGCG} 100 \mathrm{mg} / \mathrm{kg}$ (po). Both doses of EGCG protected against CM-induced renal dysfunction, as measured by serum creatinine and blood urea nitrogen (BUN). In addition, EGCG treatment markedly improved $\mathrm{CIN}$-induced oxidative stress, and resulted in a significant downregulatory effect on tumor necrosis factor (TNF)- $\alpha$ and nuclear factor (NF)- $\kappa B$ mRNA expression. Moreover, histopathological analysis showed that EGCG also attenuated CM-induced kidney damage. Considering the potential clinical use of CM and the numerous health benefits of EGCG, this study showed the protective role of multi-dose EGCG treatment on CIN and GLY-aggravated CIN through different mechanisms.
ARTICLE HISTORY

Received 11 March 2016

Accepted 20 December 2016

KEYWORDS

Contrast media;

nephrotoxicity; EGCG; rat

\section{Introduction}

With recent advances in medical technology, iodinated contrast media (ICM) has been increasingly used in radiographic diagnostic and interventional procedures. ${ }^{1,2} \mathrm{CM}$ may be nephrotoxic when combined with potentially nephrotoxic agents, or in patients with a comorbidity, such as diabetic nephropathy. The toxic effect of ICM results in acute kidney injury, and leads to a condition known as contrast-induced nephropathy (CIN). While the incidence of CIN is approximately $2 \%$ in patients without risk factors, it may range between 9 and $>50 \%$ in people with mild-to-moderate renal impairment and diabetes mellitus. ${ }^{3}$ It is now the third most common cause of hospital-acquired renal failure, and has led to increased medical care costs and increased mortality. ${ }^{1,2}$ Therefore, it is important to understand the etiology of $\mathrm{CIN}$, and to formulate effective prophylactic and therapeutic regimens to reduce its incidence. $^{3}$ Approximately 150,000 patients annually experience $\mathrm{CIN}$, and almost $1 \%$ of these individuals require dialysis and a prolonged hospital stay, with the annual cost of dialysis being estimated at $\sim \$ 32$ million. ${ }^{1}$

We used a well-established acute renal failure model with glycerol (GLY), and the CM agent iohexol, which has an improved safety and tolerability profile when compared to classic radiocontrast agents. Nevertheless, a data mining analysis of the US Food and Drug Administration databases showed that iohexol has the highest association with pooled renal events among the low osmolar contrast media (OCM) agents, including iopamidol, for which the lowest association was found. ${ }^{4}$ Therefore, iohexol has become the most interesting radiocontrast agent in $\mathrm{CIN}$-related clinical and experimental studies.

Tea (Camellia sinensis, Theaceae) is second only to water in global consumption. Green tea is one of its major forms, and represents $20 \%$ of the world's tea 
consumption. It is characterized by the presence of large amounts of catechins, ${ }^{5}$ with the major ones being epigallocatechin-3-gallate (EGCG), epicatechin-3-gallate, epigallocatechin, and epicatechin. ${ }^{6}$ Previous studies have suggested that green tea and green tea polyphenols, particularly EGCG, exert beneficial effects against neurodegenerative disease, ${ }^{7}$ cancer, ${ }^{8}$ and inflammation caused by lipid peroxidation and excessive free radical production. ${ }^{9}$ Numerous mechanisms have been proposed for the biological activities in the preventive effect of EGCG, including anti-inflammatory and antioxidant mechanisms. The protective effect of EGCG has been shown with a different underlying mechanism for pathology and protection in nephrotoxicity studies. ${ }^{10,11}$ However, there is gap in the literature, as EGCG has not been assessed with regard to the nephrotoxicity that results from CM. Therefore, this study is the first to examine the protective effect of EGCG against devastating/fatal CIN.

To our knowledge, in addition to evaluating its protective activity against different nephrotoxic agents, this study is the first to reveal the prominent protection that EGCG provides against devastating GLY-induced CM toxicity.

\section{Materials and methods}

\subsection{Animals}

A total of 48 male albino Wistar rats $(250-300 \mathrm{~g})$ obtained from Experimental Animal Laboratory of Medicinal and Experimental Application and Research Center of Atatürk University, Turkey, were used. The rats were stored on sawdust bedding in typical plastic cages in a well-ventilated room at $22^{\circ} \mathrm{C}$ under precise light conditions (14/10 h light/dark cycle), which had been approved by the local animal care committee of Atatürk University (26.06.2015/122). Standard rat food and tap water were given ad libitum.

\subsection{Reagents}

All reagents used in our laboratory experiments were purchased from the Sigma Chemical Company (Steinheim, Germany). The EGCG was a gift from DSM Nutritional Products (Istanbul, Turkey). The iohexol (OMNIPAQUE, OPAKIM Medical Products) and the GLY (Bikar Medical Products) were purchased from Istanbul, Turkey.

\subsection{Experimental design}

Eight groups were created, with six rats in each: Group 1: A healthy group

Group 2: GLY (GLY only)
Group $3 \mathrm{CM}$ (iohexol $10 \mathrm{~mL} / \mathrm{kg}$ )

Group 4: GLY + CM (iohexol $10 \mathrm{~mL} / \mathrm{kg}$ )

Group 5: CM + EGCG 50: CM (iohexol $10 \mathrm{~mL}$ / $\mathrm{kg})+\mathrm{EGCG}(50 \mathrm{mg} / \mathrm{kg}, \mathrm{po})$

Group 6: GLY + CM + EGCG 50: GLY + CM (iohexol $10 \mathrm{~mL} / \mathrm{kg})+\mathrm{EGCG}(50 \mathrm{mg} / \mathrm{kg}, \mathrm{po})$

Group 7: CM + EGCG 100: CM (iohexol $10 \mathrm{~mL}$ / $\mathrm{kg})+\mathrm{EGCG}(100 \mathrm{mg} / \mathrm{kg}, \mathrm{po})$

Group 8: GLY + CM + EGCG 100: GLY + CM (iohexol $10 \mathrm{~mL} / \mathrm{kg})+\mathrm{EGCG}(100 \mathrm{mg} / \mathrm{kg}$, po)

The EGCG doses were selected on the basis of previously published nephrotoxicity-related studies. ${ }^{12,13}$

\subsection{Contrast media-induced nephrotoxicity model}

After 24-h water deprivation, kidney injury was induced in groups 2, 4, 6, and 8 with intramuscular administration of $25 \% \mathrm{GLY}$ at a single dose of $10 \mathrm{~mL} / \mathrm{kg}$ body weight, which was divided across all legs. Drinking water and food were then resumed ad libitum. In addition to the induced kidney injury, nephrotoxicity was induced $24 \mathrm{~h}$ after GLY injection, via intravenous administration of iohexol CM into the tail vein of the animals in groups 3-8, at a single dose of $10 \mathrm{~mL} / \mathrm{kg}$ by weight over a period of $2 \mathrm{~min} .{ }^{14}$ Thirty minutes after GLY injection, the different doses of EGCG $(50-100 \mathrm{mg} / \mathrm{kg})$ was prepared in a volume of $1 \mathrm{~mL}$, dissolved in $0.9 \% \mathrm{NaCl}$, and orally administered to the animals in groups $5-8$ by gavage using an intubation needle. EGCG treatment was continued for 4 days.

Following the drug administration period, the study was completed in all groups on day 5 . Blood was collected through heart puncture deep of a general anesthetic (thiopental sodium, $50 \mathrm{mg} / \mathrm{kg}$ ), and serum was immediately separated by centrifugation at $4000 \mathrm{rpm}$ for $10 \mathrm{~min}$ at $4{ }^{\circ} \mathrm{C}$, and stored at $-80^{\circ} \mathrm{C}$ until being assayed. Kidney tissues were immediately dissected out. One kidney from each animal was stored at $-80^{\circ} \mathrm{C}$ for biochemical and molecular analyses and the remaining kidney was fixed in $10 \%$ formalin solution for pathological examination.

\subsection{Biochemical analyses}

\subsubsection{Serum measurements of blood urea nitrogen and creatinine concentration}

Serum blood urea nitrogen (BUN) (Lot No: B0382A, S.r.i, Italy) and creatinine (Lot No: B0914A, S.r.i, Italy) levels were detected using commercially available kits. All analyses were performed in a ChemWell 2910-automated-EIA and Chemistry analyzer (Awareness Technology, Inc., Palm City, FL). 


\subsubsection{Biochemical investigation of kidney tissues}

Following surgery, the tissues were stored at $-80^{\circ} \mathrm{C}$. The tissue samples from each rat were first perfused with phosphate buffered saline (PBS)/heparin and then ground in liquid nitrogen using the TissueLyser II grinding jar set (Qiagen, Hilden, Germany). Approximately $100 \mathrm{mg}$ of ground tissue was homogenized in $1 \mathrm{~mL}$ PBS homogenate buffer in an Eppendorf tube with TissueLyser II, and the samples were then centrifuged. Superoxide dismutase (SOD) activity, ${ }^{15}$ glutathione (GSH) levels, ${ }^{16}$ and malondialdehyde (MDA) levels ${ }^{17}$ from each sample supernatant and standards were measured at room temperature in duplicate via modified methods, with an enzyme-linked immunosorbent assay (ELISA) reader. A standard curve was plotted and the equation was obtained from the absorbance of the standards. The linear SOD, GSH, and MDA concentrations were calculated according to this equation, and were expressed as $\mathrm{U} / \mathrm{mg}$ protein, $\mathrm{nmol} / \mathrm{mg}$ protein, and $\mathrm{nmol} / \mathrm{mg}$ protein, respectively. The data obtained were presented as mean\pm standard deviation (SD) as $1 \mathrm{mg}$ of protein.

\subsection{Molecular investigations of kidney tissues}

\subsubsection{Total RNA extraction and CDNA synthesis}

The tissues $(20 \mathrm{mg}$ ) were stabilized in RNA stabilization reagent (RNAlater, Qiagen, Hilden, Germany), and then disrupted using the TissueLyser II $(2 \times 2$ min for kidney; $2 \times 5 \mathrm{~min}$, Qiagen). The total RNA was purified using the RNeasy Mini Kit (Qiagen) in a QIAcube (Qiagen), according to the manufacturer's instructions. The RNA samples were then reverse-transcribed into complementary DNA using the High Capacity cDNA Reverse Transcription Kit (Applied Biosystems, Foster City, CA). From $10 \mu \mathrm{L}$, total RNA was treated with $2 \mu \mathrm{L} 10 \mathrm{X}$ RT buffer, $0.8 \mu \mathrm{L} 25 \mathrm{X}$ dNTPs mix, $2 \mu \mathrm{L}$ 10X RT random primers, $1 \mu \mathrm{L}$ multiscribe reverse transcriptase, and $4.2 \mu \mathrm{L}$ DEPC- $\mathrm{H}_{2} \mathrm{O}$. Reverse transcription was carried out at $25^{\circ} \mathrm{C}$ for $10 \mathrm{~min}$, followed by $120 \mathrm{~min}$ at $37^{\circ} \mathrm{C}$, and finally $5 \mathrm{~min}$ at $85^{\circ} \mathrm{C}$, using a Veriti 96-well thermal cycler (Applied Biosystems). The concentration and quality of the CDNA were assessed and quantified using the Epoch Spectrophotometer System and Take3 Plate (BioTek Inc., Winooski, VT).

2.6.2. Relative quantification of gene expression (real-time reverse transcriptase-polymerase chain reaction)

Relative tumor necrosis factor (TNF)- $\alpha$ and nuclear factor (NF)-кB expression analyses were performed with StepOne Plus Real Time PCR System technology (Applied Biosystems, Foster City, CA) using synthesized CDNA from rat kidney RNA. A quantitative polymerase chain reaction (qPCR) was run using a TaqMan probe mix based on TaqMan probe-based technology (Applied Biosystems). A real-time $P C R$ was performed using primers generated for rat TNF- $\alpha$ Rn00562055_m1, rat NF-kB1 Rn01399583_m1, and rat $\beta$-actin Rn00667869_m1. The results are expressed as relative-fold, compared with control animals. The expression data for $\beta$-actin in each tissue were used as the endogenous control. Each determination was performed in triplicate for each tissue in a 96-well optical plate for both targets, using $9 \mu \mathrm{L}$ of cDNA (100 ng), $1 \mu \mathrm{L}$ of Primer Perfect Probe mix, and $10 \mu \mathrm{L}$ of QuantiTect Probe PCR Master mix (Qiagen) in each $20-\mu \mathrm{L}$ reaction. The plates were heated for $2 \mathrm{~min}$ at $50^{\circ} \mathrm{C}$ and then $10 \mathrm{~min}$ at $95^{\circ} \mathrm{C}$. Subsequently, 40 cycles of $15 \mathrm{~s}$ at $94^{\circ} \mathrm{C}$ and cycles of $60 \mathrm{~s}$ at $60^{\circ} \mathrm{C}$ were carried out. All data are expressed as the fold-change in expression compared to the expression in other animal groups, using the 2-delta-delta $\mathrm{Ct}\left(2^{-\Delta \Delta \mathrm{Ct}}\right)$ method. ${ }^{18}$

\subsection{Histopathological analyses}

Kidneys from the rats in all groups were obtained, sectioned frontally, and fixed in $10 \%$ neutral formalin for 48-72 $\mathrm{h}$. The tissues were then routinely processed, and embedded in paraffin wax, and 4-5- $\mu \mathrm{m}$ thick serial sections were cut. All tissue sections were stained with hematoxylin and eosin (H\&E) for histopathologic assessment, and examined under a light microscope (Olympus BX51, Japan). Tubular necrosis, hyaline casts, and hemorrhagic casts were evaluated and scored. A minimum of five fields for each kidney slide at $100 \mathrm{X}$ magnification were evaluated and assigned for severity of changes, using scores on a scale of Grade 0: (negative), Grade 1: +1 (mild), Grade 2: +2 (moderate), Grade 3: +3 (severe), and Grade 4: +4 (most severe).

\subsection{Statistical analysis}

Statistical Package for the Social Sciences (SPSS) version 20.0 (SPSS Inc., Chicago, IL) software was used for the statistical analysis. The results are presented as means $\pm S D$. Comparisons between groups were performed using one-way analysis of variance (ANOVA) and the Duncan Multiple Comparison Test. Significance was accepted at a level of $p<.05$.

\section{Results}

\subsection{Biochemical results}

\subsubsection{Serum measurements of BUN and creatinine}

The serum levels of BUN and creatinine in the control and experimental groups were evaluated. The effects of 
nephrotoxicity on serum creatinine and BUN are shown in Figures $1(A)$ and $1(B)$, respectively. BUN and creatinine serum levels significantly increased in the $\mathrm{GLY}+\mathrm{CM}$-induced nephrotoxicity compared with the other groups $(p<.05)$. EGCG administration significantly decreased the activity of the serum enzymes when compared with the GLY + CM group $(p<.05)$.

\subsubsection{Biochemical investigation of kidney tissues}

SOD activity, levels of GSH, and lipid peroxidation (MDA) were evaluated in all rat kidneys. SOD activity was significantly decreased in the GLY $+C M$ groups compared with the healthy group $(p<.05)$. In addition, significant differences between the GLY and GLY + CM groups $(p<.05)$ were found. SOD activity was significantly improved in the treatment groups compared to the GLY + CM group $(p<.05)$, as shown in Figure 2(A). GSH levels significantly were decreased in the CM and $\mathrm{GLY}+\mathrm{CM}$ group compared to the healthy group $(p<.05)$, and significant differences between the GLY and GLY + CM groups $(p<.05)$ were observed. GSH levels were significantly ameliorated in the drug treatment groups when compared to the GLY $+C M$ groups, as shown in Figure 2(B) $(p<.05)$. MDA levels were
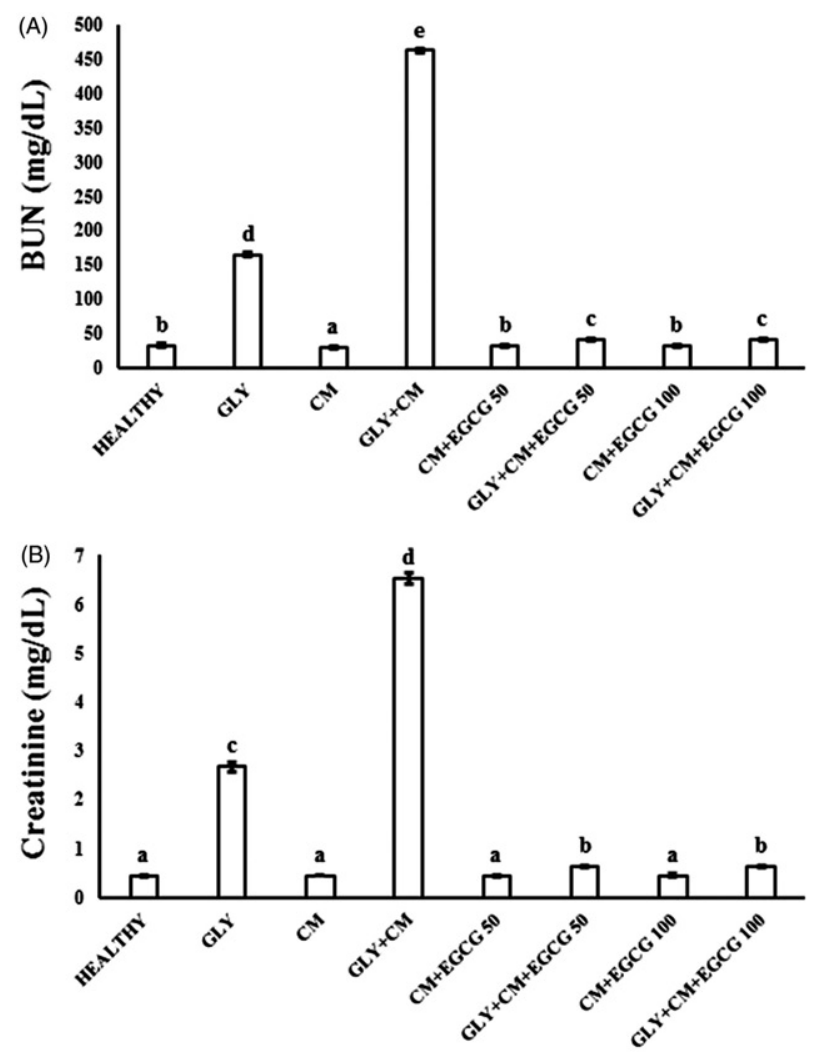

Figure 1. The serum BUN (A) and creatinine (B) levels of all the experimental groups. Means in the same column with the same letter are not significantly different; means in the same column with different letters indicate significant differences between the groups. significantly increased in the GLY + CM group compared with the healthy group $(p<.05)$, and significant differences between the GLY and GLY + CM groups $(p<.05)$ were found (Figure 2(C)). It was observed that both doses of EGCG restored MDA levels in the healthy group $(p<.05)$.

\subsection{Molecular results}

We investigated TNF- $\alpha$ and NF-KB mRNA expression in the kidney tissue of the rats using real-time PCR. As Figure 3(A) shows, TNF- $\alpha$ gene expression was 10.7-fold upregulated in the GLY $+C M$ groups compared to the healthy group $(p<.05)$. Both doses of EGCG provided a significant down-regulatory effect on TNF- $\alpha$ mRNA expression, by 10.9 -fold for the $50 \mathrm{mg} / \mathrm{kg}$ dose and
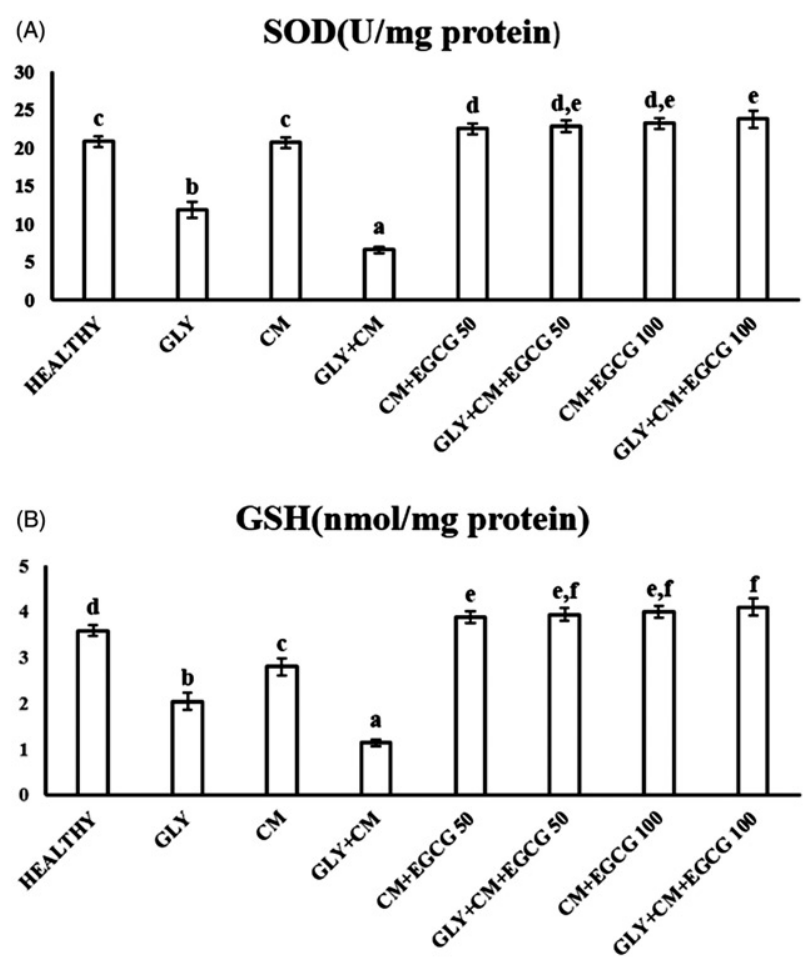

(C) $\quad$ MDA(nmol/mg protein)

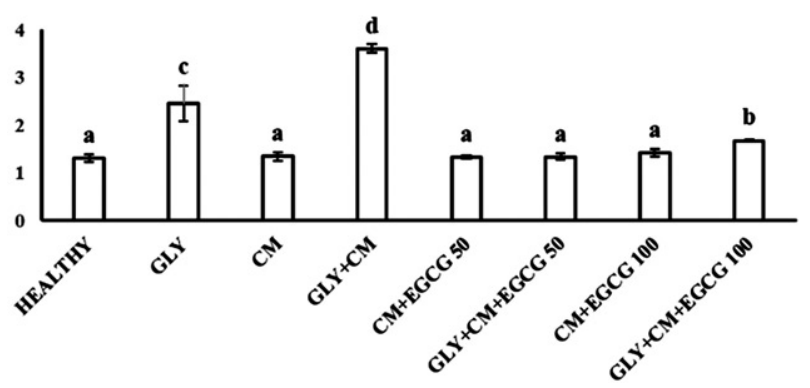

Figure 2. The tissue SOD activities (A), GSH (B) and MDA (C) levels of all the experimental groups. Means in the same column with the same letter are not significantly different; means in the same column with different letters indicate significant differences between the groups. 
11.6-fold for $100 \mathrm{mg} / \mathrm{kg}$ dose compared to GLY + CM group (both, $p<.05$ ).

The NF- $\kappa B$ mRNA level was significantly higher by 8.03-fold in the GLY + CM group compared to the control group $(p<.05)$ (Figure 3(B)). Both doses of EGCG demonstrated a significant down-regulatory effect on NF- $\kappa B$ mRNA expression in the treatment groups compared to the GLY +CM group, by 7.2-fold for the $50 \mathrm{mg} / \mathrm{kg}$ dose and 7.5 -fold for the $100 \mathrm{mg} / \mathrm{kg}$ dose compared to GLY + CM group (both, $p<.05$ ).

\subsection{Histopathological results}

Histological evaluation of kidney sections from rats in all groups cohorts subjected to radiocontrast with aggravated GLY toxicity was examined and scored hyaline and hemorrhagic casts and tubular necrosis. The results are presented in Figures 4 and 5. The control group revealed normal morphology in $100 \%$ of tubules. In the GLY group, damaged tubules were more significant than in the healthy group $(p<.05)$ and tend to increase in CM group. Hyaline and hemorrhagic casts, and necrosis in the tubules were noted (Figure 4). Degenerative changes were more evident in proximal tubules. However, hyaline and hemorrhagic casts were significantly higher in $\mathrm{CM}+\mathrm{GLY}$ group than healthy and $\mathrm{CM}$ group. The tubular necrosis was significantly
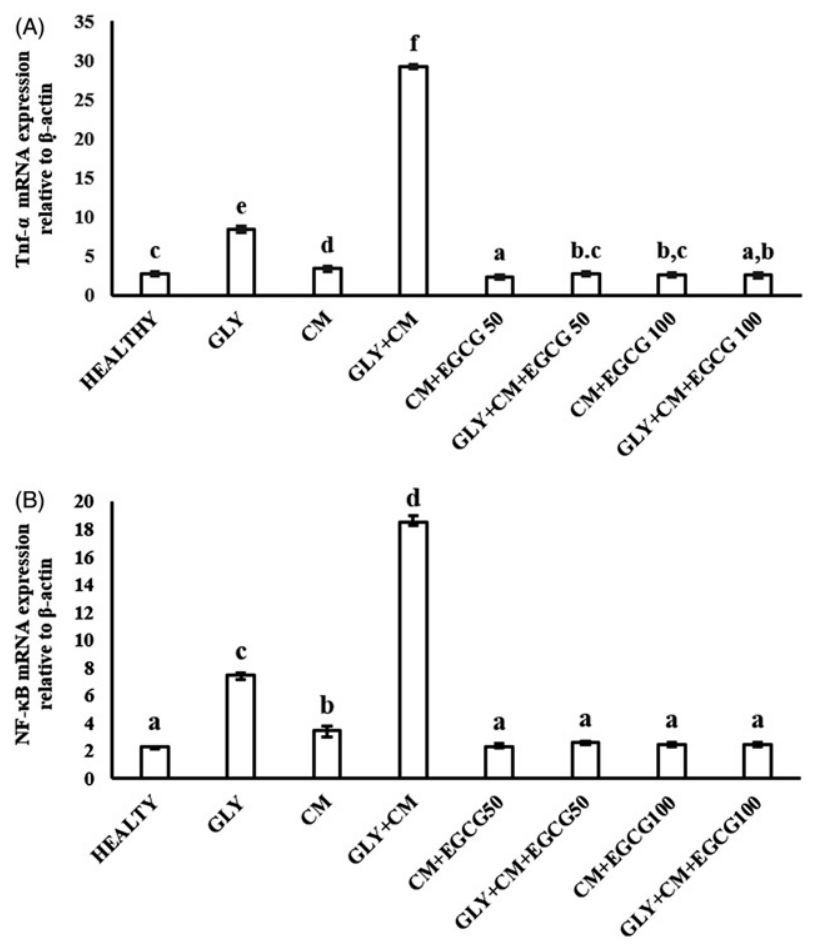

Figure 3. The tissue TNF- $\alpha(\mathrm{A})$ and NF- $\mathrm{B} B(\mathrm{~B})$ mRNA expressions of all the experimental groups. Means in the same column with the same letter are not significantly different; means in the same column with different letters indicate significant differences between the groups. increased in group $\mathrm{CM}+\mathrm{GLY}$ in comparison with $\mathrm{CM}$ group. The treatment with EGCG before radiocontrast application significantly improved renal morphology compared with the CM+GLY groups $(p<.05)$, but some tubules with edema and vacuolization remained (Figure 5). To a certain extent, acute tubular necrosis developed frequently in the $\mathrm{CM}+\mathrm{GLY}$ compared with the $\mathrm{CM}+\mathrm{GLY}+\mathrm{EGCG}$ groups. However, the hyaline and hemorrhagic casts were not decreased as much as the control and CM levels.

\section{Discussion}

This study investigated the protective role of the natural compound EGCG at two different doses (50 and $100 \mathrm{mg} / \mathrm{kg}$ ) in CIN and CIN aggravated by GLY. We showed that EGCG reduced the increased levels of serum BUN and creatinine in rats in GLY-aggravated CIN. Furthermore, EGCG provided significant protection against increased oxidative stress and inflammation due to nephrotoxicity through NF- $\kappa B$ and TNF- $\alpha$ mRNA expression levels. In addition, histopathology showed that EGCG ameliorated the renal injury. Considering the potential clinical use of $\mathrm{CM}$ and the numerous health benefits of EGCG, this study was undertaken to assess the protective role of multi-dose EGCG treatment on CIN and GLY-aggravated CIN. Elevation of the serum level of creatinine after exposure to a contrast agent is most frequently used in diagnosing CIN. Filtration between the renal tubules and glomerules is represented by serum levels of creatinine. ${ }^{1}$ In clinical practice, level of serum creatinine, which is a surrogate marker of glomerular filtration rate, has been used for the diagnosis of $\mathrm{CIN}^{2}$ Increased serum BUN and creatinine levels have previously been reported in several $\mathrm{CIN}$ studies. ${ }^{19}$ The ameliorative effect of EGCG on BUN and creatinine levels has also previously been shown. It was found that BUN and creatinine decreased with EGCG treatment in the presence of various nephrotoxic drugs, such as cyclosporine and cisplatin. ${ }^{10-12}$ In accordance with previous studies, we found that EGCG treatment decreased BUN and creatinine levels, which are increased in CIN and GLY-aggravated CIN, thereby indicating improved renal function. Both doses of EGCG had the same effect on BUN and creatinine levels.

The pathogenesis of CIN is not clear, but recent studies have shown that it is caused by a combination of toxic and ischemic injury to the renal tubular cells. The risk of CIN has decreased with the use of low-osmolar agents, such as iohexol. ${ }^{1} \mathrm{CM}$ reduces blood flow through the kidney areas, and increases hypoxic damage. $^{2}$ Direct cytotoxic effects, auto- and paracrine factors that perturb renal hemodynamics, altered 


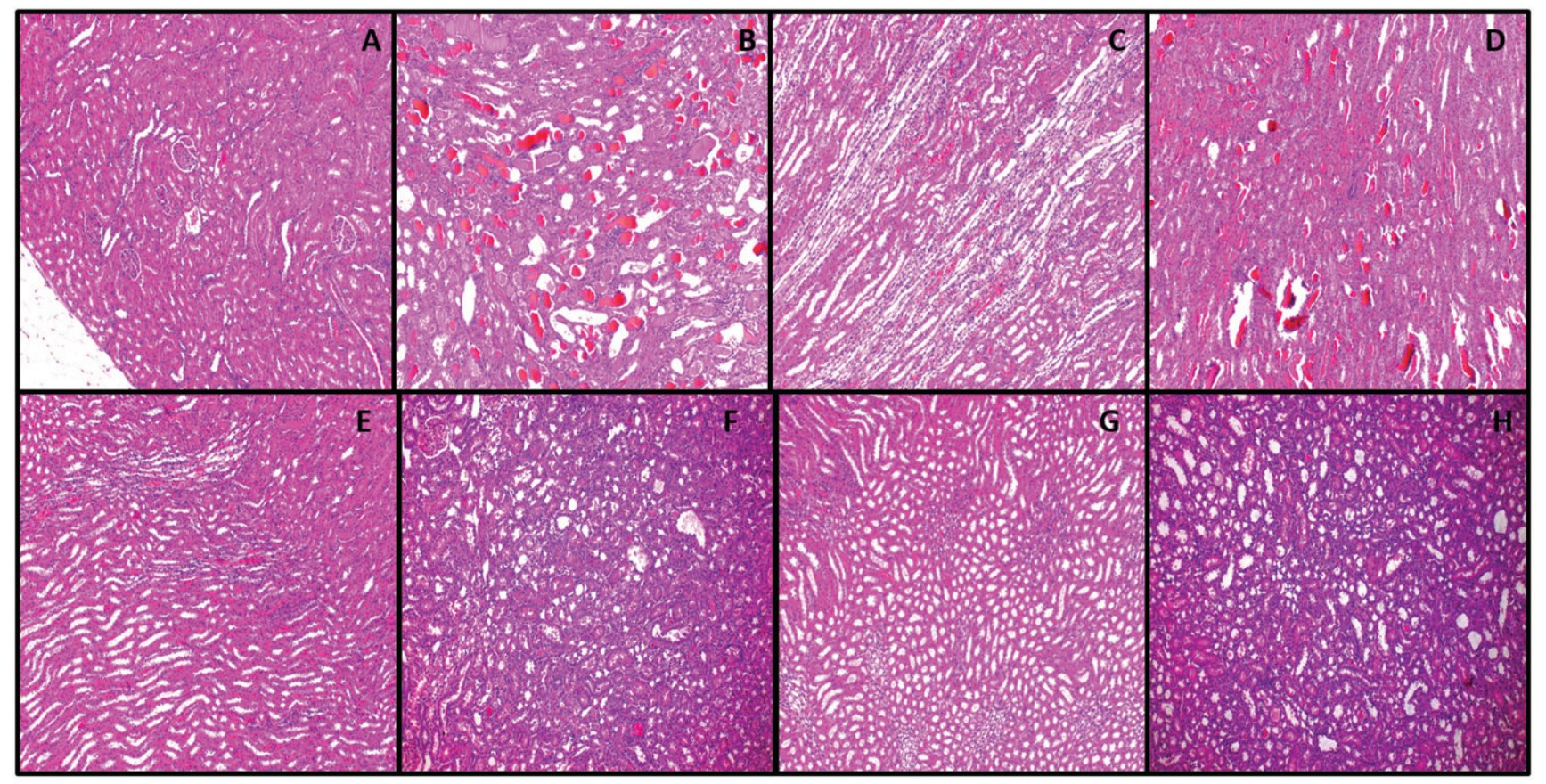

Figure 4. Hematoxylin and eosin results in rats' kidney tissues; magnification 100X. A: Healthy B: GLY C: CM D: GLY+CM E: CM + EGCG 50 F: GLY + CM + EGCG 50 G: CM + EGCG 100 H: GLY + CM + EGCG 100

rheological properties, which affect renal hemodynamics and tubulodynamics, and regional hypoxia are the mechanisms underlying $\mathrm{CIN}$ and these act in concert. ${ }^{2}$ However, the clinical features and the pathophysiological mechanism of $\mathrm{CIN}$, alone or in the presence of other underlying renal pathologies, are significantly differentiated. $^{20}$ In our study, pretreatment with GLY was used to induce preexisting renal insufficiency and mimic any underlying pathology. In GLY-induced renal failure, ischemic injury or tubular nephrotoxicity occurs. ${ }^{21}$

Protection of the renal tubules from prolonged contact with $\mathrm{CM}$ is the goal of prevention, as permanent damage can occur at the time of contact. Sodium bicarbonate and $\mathrm{N}$-acetyl cysteine (NAC) are used to prevent an acidic environment and formation of free radicals in the renal tubules. Antioxidant protection preserves at the level of the glomerulus and reduces level of systemic inflammatory factors. ${ }^{1}$ Although some prevention strategies exist, including adequate hydration of the patient, discontinuation of nephrotoxic drugs before $C M$ administration and NAC, vitamins $C$ and $E$ treatment, ${ }^{22}$ and some compounds such as infliximab ${ }^{23}$ and simvastatin ${ }^{24}$ have been shown to ameliorate $\mathrm{CIN}$; there is no available standard prophylactic or therapeutic agent for this condition.

EGCG is the most abundant catechin, and represents $50-80 \%$ of catechins by weight. ${ }^{5,6}$ Its ability to quench free radical species, including the hydroxyl radical, superoxide anion, nitric oxide, and peroxynitrite, and to chelate transition metals, means that EGCG has strong antioxidant activity. ${ }^{5}$ Under optimal dose regimens, both properties of EGCG are expected to contribute to the modulation of the oxidative stress response. Although exposure to a low concentration of a pro-oxidant prior to exposure to oxidative stress induces adaptation, exposure to an antioxidant concurrently with exposure to the oxidative stress affords protection against the oxidative stress. ${ }^{25}$ Tea polyphenols, particularly EGCG, have generally been regarded as antioxidants, and the emerging evidence for the protective effects of this compound is interesting and raises numerous potential questions.

Oxidative stress, the perturbation of the balance between pro-oxidants and antioxidants, may underline the pathology of $\mathrm{CIN} .^{23,26}$ EGCG has antioxidant activity, which is attributed to the galloyl group at the 3 position and the o-trihydroxyl group in the B ring in its structure, and it protects cells from damage by reactive oxygen species (ROS). Thus, EGCG may have an antioxidant effect on oxidative stress-induced impaired renal function. $^{10,13}$ In our study, administration of GLY and $\mathrm{GLY}+\mathrm{CM}$ resulted in depletion of enzymatic and nonenzymatic antioxidant defense systems, leading to a significant decrease in SOD activity and GSH levels. Although these antioxidant levels significantly changed in the GLY-aggravated CIN group, when compared to the GLY group, treatment with CM did not change SOD activity but changed GSH levels. We report that both doses of EGCG $(50$ and $100 \mathrm{mg} / \mathrm{kg})$ significantly increased SOD activity and GSH levels when compared 


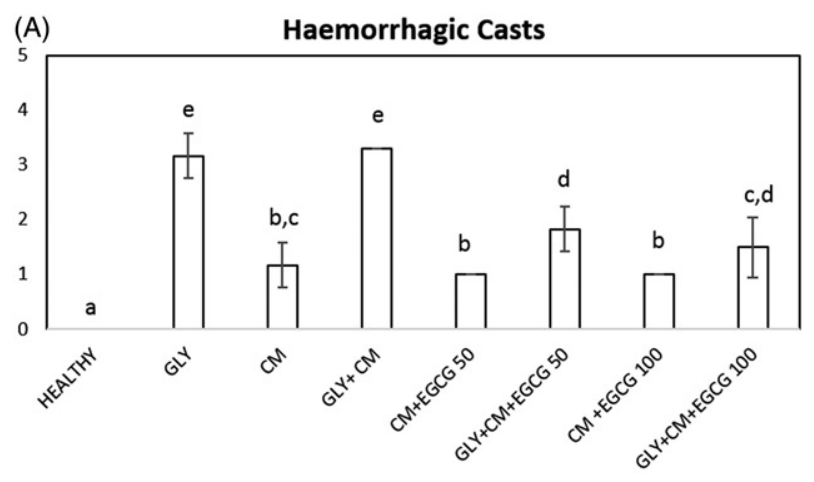

(B) Tubuler Casts

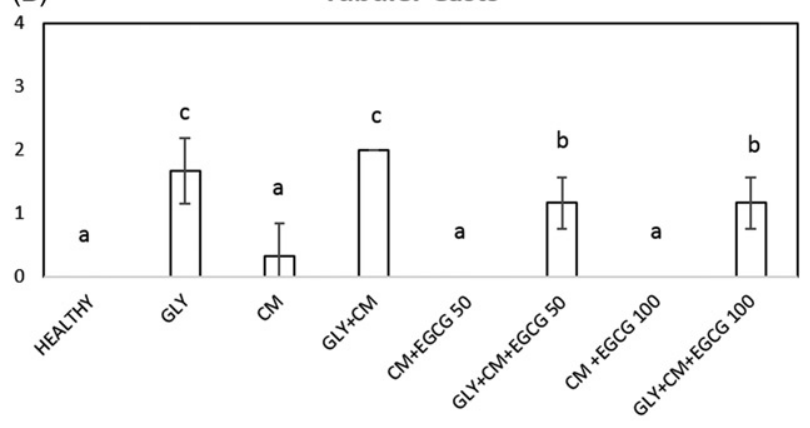

(C) Hyaline Casts

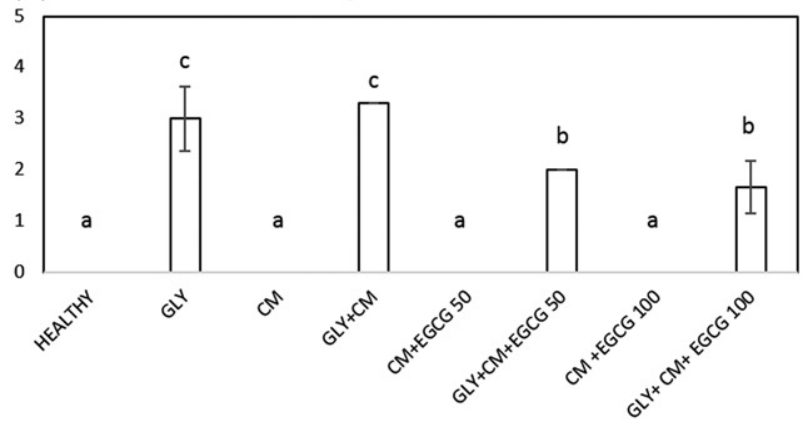

Figure 5. Effects of EGCG on histopathological hemorrhagic cast (A) tubular casts (B) and hyaline cast (C) scores in rats' kidneys. Means in the same column by the same letter are not significantly different from the test of Duncan $(p=.05)$. Results are expressed as means \pm SD $(n=6)$.

to the control group. The effect of EGCG did not change, irrespective of the dose administered, but SOD activity and GSH levels approached the GSH levels and SOD activity of the control group at both doses. Decreased SOD activity and GSH levels have previously been reported in $\mathrm{CIN}$, and different treatment strategies have ameliorated oxidative stress. ${ }^{23,26}$ In accordance with the results of our study, Saritemur et al. showed decreased SOD activity and GSH levels in GLY-aggravated $\mathrm{CIN}$, and treatment with infliximab increased SOD activity and GSH levels. ${ }^{23}$

Oxygen free radicals lead to peroxidation of lipids and produce damage to cells and tissues, which may be another mechanism of $\mathrm{CIN} .{ }^{10}$ MDA levels increased to statistically significant levels in the GLY $+C M$ group compared with the control group in this study, while lipid peroxidation also increased as a result of oxidative stress in the GLY treatment group, but not in the CM treatment groups. Both doses of EGCG decreased the MDA levels, but only the $50 \mathrm{mg} / \mathrm{kg}$ dose reduced the MDA levels to control levels. These results reveal that CM increased nephrotoxicity in the presence of kidney injury via an oxidative stress mechanism, and that consumption of EGCG ameliorates this effect with its radical-scavenging activity. These data are in agreement with previous studies conducted with various antioxidant supplements. In the light of these oxidative stress parameters results, we can state that $\mathrm{CM}$ alone did not affect oxidative stress, but it caused nephrotoxicity via the oxidative stress mechanism in the presence of kidney pathology. In addition, treatment with EGCG can block this effect via its strong antioxidant effect.

ROS are known to enhance inflammation through the activation of NF- $\kappa B$ via phosphorylation of $\mathrm{I}-\mathrm{\kappa B}$. Stimuli that activate NF- $\kappa B$ also induce oxidative stress, and some evidence has shown that NF- $\kappa B$ activation is stimulated by ROS and is inhibited by antioxidants. Antioxidants can suppress the NF- $\kappa B$ activation that is induced by $\mathrm{ROS}^{27}$ and EGCG has been shown to inhibit the constitutive activation of NF- $\mathrm{KB}$ in different cell culture studies. ${ }^{28}$ Pan et al. suggested that EGCG might influence the inflammatory response in cisplatin nephrotoxicity through the NF-KB pathway. ${ }^{29}$ In another study, Sahin et al. also showed the preventive effect of EGCG against cisplatin-induced NF- $\kappa B$ expression in rat kidney. ${ }^{12}$ In accordance with these studies, NF- $\kappa B$ mRNA expression was up-regulated with GLY, CM, and $\mathrm{GLY}+\mathrm{CM}$ administrations. The highest expression was found in the GLY + CM group, showing that $C M$ is more potent in kidney injury in the presence of renal failure. Both doses of EGCG treatment diminished the increased mRNA expression of NF- $\mathrm{KB}$ in the GLY, CM, and $\mathrm{GLY}+\mathrm{CM}$ groups to control group values. Therefore, EGCG is beneficial in nephrotoxicity.

The production of pro-inflammatory cytokines, such as TNF- $\alpha$, is increased in the inflammatory process, which is another factor that plays a role in the pathogenesis of nephrotoxicity. ${ }^{30}$ Increased TNF- $\alpha$ levels were observed in a study conducted on cisplatin-induced nephrotoxicity. ${ }^{13}$ El-Mowafy et al. evaluated the protective effect of EGCG in cisplatin-induced kidney injury, via the role of inflammatory cytokines. Although TNF- $\alpha$ levels increased in kidney homogenates in the cisplatin treatment groups, treatment with $50 \mathrm{mg} / \mathrm{kg}$ EGCG significantly decreased these levels. ${ }^{13}$ Saritemur et al. showed the mRNA expression of TNF- $\alpha$ in GLYaggravated radiocontrast nephrotoxicity in rats, and that this was decreased by the TNF- $\alpha$ inhibitor 
infliximab. ${ }^{23}$ Consistent with these findings, TNF- $\alpha$ mRNA expression increased in the rats that were administered GLY, CM, or GLY + CM in this study, and these levels decreased in the rats that were administered 50 and $100 \mathrm{mg} / \mathrm{kg}$ EGCG.

Tubular necrosis, tubular epithelial damage, hyaline casts, and hemorrhagic casts have been found in various studies, ${ }^{31}$ and it has been shown that tubular necrosis and epithelial injury are significant indicators in nephrotoxicity injury in these studies. ${ }^{32}$ In association with the tubular injury, significant damage to hemorrhagic renal tubules occurs with hyaline in the development of nephropathy. In our histopathological studies, we determined the ameliorative effect of both EGCG doses on tubular structure, but the changes did not differ between doses.

\section{Conclusions}

In conclusion, we have demonstrated that (1) the administration of EGCG attenuates kidney injury and increases antioxidant enzymes; (2) the administration of EGCG significantly inhibits inflammation mediated by $\mathrm{NF}-\kappa \mathrm{B}$ and TNF- $\alpha$ in rats with GLY-aggravated kidney injury. Further clinical investigation is needed to better clarify the usefulness of EGCG in CIN in clinic.

\section{Acknowledgments}

The authors thank to the DSM Nutritional Products (Istanbul, Turkey) for providing EGCG (Teavigo).

\section{Disclosure statement}

The authors report no conflicts of interest. The authors alone are responsible for the content and writing of the paper.

\section{References}

1. Jorgensen AL. Contrast-induced nephropathy: Pathophysiology and preventive strategies. Crit Care Nurse. 2013;33:37-46.

2. Seeliger $E$, Sendeski M, Rihal CS, Persson PB. Contrastinduced kidney injury: Mechanisms, risk factors, and prevention. Eur Heart J. 2012;33:2007-2015.

3. Sadat U. Radiographic contrast-media-induced acute kidney injury: Pathophysiology and prophylactic strategies. ISRN Radiol. 2013;2013:496438.

4. Solomon R, Dumouchel W. Contrast media and nephropathy: Findings from systematic analysis and Food and Drug Administration reports of adverse effects. Invest Radiol. 2006;41:651-660.

5. Lambert JD, Elias RJ. The antioxidant and pro-oxidant activities of green tea polyphenols: A role in cancer prevention. Arch Biochem Biophys. 2010;501:65-72.
6. Singh BN, Shankar S, Srivastava RK. Green tea catechin, epigallocatechin-3-gallate (EGCG): Mechanisms, perspectives and clinical applications. Biochem Pharmacol. 2011;82:1807-1821.

7. Walker JM, Klakotskaia D, Ajit D, et al. Beneficial effects of dietary EGCG and voluntary exercise on behavior in an Alzheimer's disease mouse model. J Alzheimers Dis. 2015;44:561-572.

8. Mayr C, Wagner A, Neureiter D, et al. The green tea catechin epigallocatechin gallate induces cell cycle arrest and shows potential synergism with cisplatin in biliary tract cancer cells. BMC Complement Altern Med. 2015;15:194.

9. Shen $K$, Feng $X$, Su R, Xie $H$, Zhou $L$, Zheng $S$. Epigallocatechin 3-gallate ameliorates bile duct ligation induced liver injury in mice by modulation of mitochondrial oxidative stress and inflammation. PLoS One. 2015;10:e0126278.

10. Chang EJ, Mun KC. Effect of epigallocatechin gallate on renal function in cyclosporine-induced nephrotoxicity. Transplant Proc. 2004;36:2133-2134.

11. Fatima S, Al-Mohaimeed N, Arjumand S, Banu N, AlJameil N, Al-Shaikh Y. Effect of pre- and post-combined multidoses of epigallocatechin gallate and coenzyme Q10 on cisplatin-induced oxidative stress in rat kidney. J Biochem Mol Toxicol. 2015;29:91-97.

12. Sahin K, Tuzcu M, Gencoglu $H$, et al. Epigallocatechin3-gallate activates $\mathrm{Nrf2/HO}-1$ signaling pathway in cisplatin-induced nephrotoxicity in rats. Life Sci. 2010;87:240-245.

13. El-Mowafy AM, Salem HA, Al-Gayyar MM, El-Mesery ME, El-Azab MF. Evaluation of renal protective effects of the green-tea (EGCG) and red grape resveratrol: Role of oxidative stress and inflammatory cytokines. Nat Prod Res. 2011;25:850-856.

14. Duan SB, Liu FY, Luo JA, et al. Nephrotoxicity of highand low-osmolar contrast media. The protective role of amlodipine in a rat model. Acta Radiol. 2000; 41:503-507.

15. Sun Y, Oberley LW, Li Y. A simple method for clinical assay of superoxide dismutase. Clin Chem. 1988;34:497-500.

16. Sedlak J, Lindsay RH. Estimation of total, proteinbound, and nonprotein sulfhydryl groups in tissue with Ellman's reagent. Anal Biochem. 1968;25:192-205.

17. Ohkawa H, Ohishi N, Yagi K. Assay for lipid peroxides in animal tissues by thiobarbituric acid reaction. Anal Biochem. 1979;95:351-358.

18. Livak KJ, Schmittgen TD. Analysis of relative gene expression data using real-time quantitative PCR and the 2(-delta delta C(T)) method. Methods. 2001;25: 402-408.

19. Ozgur T, Tutanc M, Zararsiz I, et al. The protective effect of ebselen on radiocontrast-induced nephrotoxicity. Ren Fail. 2012;34:991-997.

20. Bartholomew BA, Harjai KJ, Dukkipati S, et al. Impact of nephropathy after percutaneous coronary intervention and a method for risk stratification. Am J Cardiol. 2004;93:1515-1519.

21. Singh $A P$, Junemann $A$, Muthuraman $A$, et al. Animal models of acute renal failure. Pharmacol Rep. 2012;64:31-44. 
22. Andreucci $M$, Solomon $R$, Tasanarong $A$. Side effects of radiographic contrast media: Pathogenesis, risk factors, and prevention. Biomed Res Int. 2014;2014:741018.

23. Saritemur M, Un H, Cadirci E, et al. Tnf-alpha inhibition by infliximab as a new target for the prevention of glycerol-contrast-induced nephropathy. Environ Toxicol Pharmacol. 2015;39:577-588.

24. Al-Otaibi KE, Al Elaiwi AM, Tariq M, Al-Asmari AK. Simvastatin attenuates contrast-induced nephropathy through modulation of oxidative stress, proinflammatory myeloperoxidase, and nitric oxide. Oxid Med Cell Longev. 2012;2012:831748.

25. Yiannakopoulou E. Targeting oxidative stress response by green tea polyphenols: Clinical implications. Free Radic Res. 2013;47:667-671.

26. Kunak CS, Ugan RA, Cadirci E, et al. Nephroprotective potential of carnitine against glycerol and contrastinduced kidney injury in rats through modulation of oxidative stress, proinflammatory cytokines, and apoptosis. Br J Radiol. 2016;89:20140724.
27. Higdon JV, Frei B. Tea catechins and polyphenols: Health effects, metabolism, and antioxidant functions. Crit Rev Food Sci Nutr. 2003;43:89-143.

28. Yang CS, Sang S, Lambert JD, Hou Z, Ju J, Lu G. Possible mechanisms of the cancer-preventive activities of green tea. Mol Nutr Food Res. 2006;50:170-175.

29. Pan H, Chen J, Shen K, et al. Mitochondrial modulation by epigallocatechin 3-gallate ameliorates cisplatin induced renal injury through decreasing oxidative/ nitrative stress, inflammation and NF-kB in mice. PLoS One. 2015;10:e0124775.

30. Miller RP, Tadagavadi RK, Ramesh G, Reeves WB. Mechanisms of cisplatin nephrotoxicity. Toxins (Basel). 2010;2:2490-2518

31. Droguett $A$, Krall $P$, Burgos $M E$, et al. Tubular overexpression of gremlin induces renal damage susceptibility in mice. PLoS One. 2014;9:e101879.

32. Naziroglu M, Yoldas N, Uzgur EN, Kayan M. Role of contrast media on oxidative stress, $\mathrm{Ca}(2+)$ signaling and apoptosis in kidney. J Membr Biol. 2013;246:91-100. 